\title{
Differences in Urodynamic Parameters According to the Presence of a Hunner Lesion in Women With Interstitial Cystitis/Bladder Pain Syndrome
}

Sun Tae Ahn, Hyeong Guk Jeong, Tae Yong Park, Jong Wook Kim, Hong Seok Park, Du Geon Moon, Jeong Gu Lee, Mi Mi Oh

Department of Urology, Korea University College of Medicine, Seoul, Korea

Purpose: Differences in the severity of subjective symptoms have been noted depending on whether a Hunner lesion is present in women with interstitial cystitis/bladder pain syndrome (IC/BPS). In this study, we aimed to identify differences in objective urodynamic parameters in women with IC/BPS according to the presence of a Hunner lesion.

Methods: This cross-sectional study included a total of 55 patients with IC/BPS. IC/BPS and the presence of a Hunner lesion on cystoscopy were diagnosed according to American Urological Association guidelines. The patients were categorized into a Hunner IC/BPS group and a non-Hunner IC/BPS group according to the presence of a Hunner lesion on cystoscopy. At the initial visit, a medical history was taken from all patients with IC/BPS, and they underwent symptom assessment using a 3-day voiding diary and laboratory tests. A urodynamic study was then performed before any treatment was performed. Baseline characteristics and urodynamic parameters were compared between the 2 groups.

Results: Of the 55 patients, 23 (41.8\%) had a Hunner lesion on cystoscopy. As documented in the voiding diaries, the Hunner IC/BPS group had more frequent voids and a smaller maximal voided volume $(\mathrm{P}=0.045, \mathrm{P}<0.001$, respectively). Regarding urodynamic parameters, the mean volume at the first desire to void, normal desire to void, strong desire to void (SDV), and maximum cystometric bladder capacity $(\mathrm{MBC})$ was significantly lower in the Hunner IC/BPS group $(\mathrm{P}=0.001, \mathrm{P}=0.004$, $\mathrm{P}<0.001$, and $\mathrm{P}<0.001$, respectively). On receiver operating characteristic curve analysis, patients with an $\mathrm{SDV} \leq 210 \mathrm{~mL}(\operatorname{area}$ under the curve $[\mathrm{AUC}]=0.838, \mathrm{P}<0.001)$ and an $\mathrm{MBC} \leq 234 \mathrm{~mL}(\mathrm{AUC}=0.857, \mathrm{P}<0.001)$ were likely to be in the Hunner IC/ BPS group.

Conclusions: The differences in patients' subjective symptoms between the Hunner IC/BPS and non-Hunner IC/BPS groups were confirmed to correspond to differences in objective urodynamic parameters.

Keywords: Interstitial Cystitis; Bladder Pain Syndrome; Hunner Lesion; Urodynamic Parameters

- Research Ethics: The study protocol was reviewed and approved by the Institutional Review Board (IRB) of Korea University Hospital (2016GR0144). Written informed consent by the patients was waived by the IRB due to a retrospective nature of our study.

- Conflict of Interest: MMO, a member of the Editorial Board of INJ, is the corresponding author of this article. However, she played no role whatsoever in the editorial evaluation of this article or the decision to publish it. No potential conflict of interest relevant to this article was reported.

\section{INTRODUCTION}

Interstitial cystitis/bladder pain syndrome (IC/BPS) is a clinical diagnosis based on symptoms of urgency, urinary frequency, and pelvic and/or bladder pain in the absence of identifiable pathology [1,2]. As IC/BPS represents a symptom complex with
Corresponding author: $\mathrm{Mi} \mathrm{Mi} \mathrm{Oh} \mathrm{(iD} \mathrm{https://orcid.org/0000-0002-1232-2598}$ Department of Urology, Korea University Guro Hospital, Korea University College of Medicine, 148 Gurodong-ro, Guro-gu, Seoul 08308, Korea E-mail: mamah@hanmail.net / Tel: +82-2-2626-3203 / Fax: +82-2-2626-1321 Submitted: December 1, 2017 / Accepted after revision: January 11, 2018
This is an Open Access article distributed under the terms of the Creative Commons Attribution Non-Commercial License (http://creativecommons.org/licenses/by-nc/4.0/) which permits unrestricted non-commercial use, distribution, and reproduction in any medium, provided the original work is properly cited. 
a wide spectrum of symptoms, the wish to include all of them into a single entity has resulted in much confusion in terminology and missed diagnoses [3]. In this context, the European Society for the Study of Interstitial Cystitis (ESSIC) proposed a classification of IC/BPS according to the findings of cystoscopy with hydrodistention and morphological findings in bladder biopsies [4].

Patients with a Hunner lesion are classified as having type 3 IC/BPS. Hunner lesion is the most distinct cystoscopic finding in IC/BPS, with characteristic central fragility and the presence of a deep rupture through the mucosa and submucosa when bladder distention is provoked [5]. Although the ESSIC classifies IC/BPS patients according to cystoscopic findings, there has been a long debate on the clinical significance of the different IC/BPS subtypes [6]. However, recent studies reported that patients with a Hunner lesion have more severe clinical symptoms, with more frequent voiding and a lower bladder capacity than patients without a Hunner lesion $[7,8]$. Unfortunately, these findings do not provide guidance regarding which patients should undergo cystoscopy to identify whether a Hunner lesion is present, since the symptoms of the patients are subjective, not objective results from a test. These subjective differences need to be confirmed objectively. Therefore, we conducted this study, in which we compared urodynamic parameters in patients with IC/BPS according to the presence of a Hunner lesion, in order to establish objective differences between IC/BPS with a Hunner lesion and IC/BPS without a Hunner lesion.

\section{MATERIALS AND METHODS}

A cross-sectional and retrospective study was performed to investigate the urodynamic data obtained from patients between June 2010 and June 2012. The study protocol was reviewed and approved by the Institutional Review Board (IRB) of Korea University Hospital (2016GR0144). Written informed consent by the patients was waived by the IRB due to a retrospective nature of our study. During this period, 55 female patients with IC/BPS were identified. The diagnosis of IC/BPS was based on the definition contained in the American Urological Association guidelines (an unpleasant sensation perceived to be related to the urinary ladder, associated with lower urinary tract symptoms of more than 6 weeks' duration, in the absence of infection or other identifiable causes) [9].

A comprehensive medical history was obtained, including age, symptom duration, medical history, history of gynecologi- cal or urological surgery, body mass index, menopause status, parity, and history of stress urinary incontinence. All patients underwent a physical examination and completed a 3-day voiding diary. Urinalysis, urinary cultures, and urinary cytology were also performed in all patients to exclude urinary tract infections and bladder cancer.

A urodynamic study (UDS) was performed in patients with severe lower urinary symptoms refractory to conventional treatment. Among these patients, we selected those who were diagnosed with IC/BPS and retrospectively reviewed their records in this study. The UDS was performed on an outpatient basis before any treatment in all patients. All patients agreed to stop taking medications that could influence the test results for 2 weeks before the UDS, including alpha-adrenergic blocking agents, anticholinergic drugs, pentosane polysulfate, and tricyclic antidepressants. The UDS was performed according to the standard International Continence Society protocol by 1 female examiner in an identically aseptic manner [1]. Before the UDS, all patients underwent uroflowmetry, with a measurement of post-void residual urine, and completely emptied via catheterization. Before starting the filling cystometry, the examiner explained the 3 normal bladder sensations to the patients: first desire to void (FDV), normal desire to void (NDV), and strong desire to void (SDV). The maximum cystometric bladder capacity (MBC) was measured, and was defined as the point at which patients could not endure the pain or strong impulse/ SDV any longer.

After completing the examinations in an outpatient setting, the patients were admitted and cystoscopy and hydrodistention were performed under general anesthesia to identify the presence of a Hunner lesion and to perform fulguration simultaneously. The presence of a Hunner lesion on cystoscopy was diagnosed based on the American Urological Association guidelines. The patients were categorized into a Hunner IC/BPS group and a non-Hunner IC/BPS group according to the presence of a Hunner lesion on cystoscopy.

The differences in the baseline characteristics and urodynamic parameters between the Hunner IC/BPS and non-Hunner IC/BPS groups were analyzed. Categorical variables were examined using the Pearson chi-square test where appropriate (expected frequency $>5$ ), and otherwise the Fisher exact test was used. Continuous variables are expressed as mean \pm standard deviation, and were examined using the Mann-Whitney U-test (nonparametric analysis of variance). Additionally, receiver operating characteristic (ROC) analysis was used to measure the 
value of the parameters that we measured as diagnostic tools. All analyses were performed using IBM SPSS Statistics ver. 22.0 (IBM Co., Armonk, NY, USA). P-values $<0.05$ were considered to indicate statistical significance.

\section{RESULTS}

Of the 55 patients, 23 (41.8\%) had a Hunner lesion, and glomerulations were found in 47 (85.5\%) on cystoscopy. The demographic and clinical characteristics of the Hunner IC/BPS group and the non-Hunner IC/BPS group are presented in Table 1. Age, symptom duration, menopausal status, and history of pelvic surgery were similar between the groups. In the analysis of the voiding diaries, patients with a Hunner lesion had more frequent voids and a smaller maximal voided volume (MVV), whereas no significant differences were observed in the number of episodes of urge incontinence or the minimal voided volume.

Regarding urodynamic parameters, the mean volumes of FDV, NDV, SDV, and MBC on filling cystometry were significantly lower in the Hunner IC/BPS group than in the nonHunner IC/BPS group $(\mathrm{P}=0.001, \mathrm{P}=0.004, \mathrm{P}<0.001$, and $\mathrm{P}<0.001$, respectively). Additionally, the bladder compliance was normal in both groups, but was significantly lower in the Hunner IC/BPS group than in the non-Hunner IC/BPS group $\left(24.0 \pm 12.8 \mathrm{~mL} / \mathrm{cm} \mathrm{H}_{2} \mathrm{O}\right.$ vs. $\left.30.3 \pm 9.7 \mathrm{~mL} / \mathrm{cm} \mathrm{H}_{2} \mathrm{O}, \mathrm{P}=0.035\right)$. There was no significant gap between the MVV and MBC in either group, and the volume discrepancy between the MVV and $\mathrm{MBC}$ was not significantly different between the 2 groups (Table 2).

In the ROC curve analysis to identify the potential criteria for static urodynamic parameters predictive of the presence of a Hunner lesion, the SDV and MBC showed an area under the curve of 0.838 (95\% confidence interval [CI], 0.73-0.95) and 0.857 (95\% CI, 0.74-0.96), respectively ( $\mathrm{P}<0.001$, both) (Fig. 1). The optimal cutoff values to maximize the Youden index [10] were $210 \mathrm{~mL}$ for the SDV and $236 \mathrm{~mL}$ for the MBC (Youden index $=0.584$ and 0.670 , respectively). Using this cutoff value of $210 \mathrm{~mL}$ for the SDV, the sensitivity and specificity were 95.5\% and 63.0\%, respectively. Additionally, at this volume of the SDV, the positive predictive value (PPV) and negative predictive value (NPV) were $67.7 \%$ and $94.4 \%$, respectively. Using the cutoff value of $236 \mathrm{~mL}$ for the $\mathrm{MBC}$, the sensitivity and specificity were $82.6 \%$ and $84.4 \%$, respectively. At this value of $236 \mathrm{~mL}$ for the MBC, the PPV and NPV were $79.2 \%$ and $87.1 \%$, respectively.

Table 1. Baseline characteristics and voiding diary parameters in patients with and without a Hunner lesion

\begin{tabular}{lccc}
\hline Characteristic & Non-Hunner IC/BPS $(\mathrm{n}=32)$ & Hunner IC/BPS $(\mathrm{n}=23)$ & P-value \\
\hline Age $(\mathrm{yr})$ & $54.91 \pm 13.02$ & $46.70 \pm 14.47$ & $0.054^{\mathrm{a})}$ \\
Symptom duration $(\mathrm{mo})$ & $19.78 \pm 28.057$ & $29 \pm 40.430$ & $0.331^{\mathrm{a})}$ \\
Body mass index $\left(\mathrm{kg} / \mathrm{m}^{2}\right)$ & $22.457 \pm 2.67$ & $22.262 \pm 3.596$ & $0.828^{\mathrm{a})}$ \\
Menopause & $19(59.4)$ & $8(34.8)$ & $0.074^{\mathrm{b})}$ \\
No. of vaginal deliveries & $2.0 \pm 0.9$ & $1.6 \pm 1.1$ & $0.082^{\mathrm{a})}$ \\
Pelvic surgery & $13(40.6)$ & $5(21.7)$ & $0.141^{\mathrm{b})}$ \\
Stress urinary incontinence & $7(21.9)$ & $5(21.7)$ & $0.990^{\mathrm{b})}$ \\
Cystoscopy & & & \\
Glomerulation & 27 & 20 & $0.553^{\mathrm{a})}$ \\
Cystoscopic MBC $(\mathrm{mL})$ & $406.2 \pm 105.1$ & $284.4 \pm 102.6$ & $<0.001^{\mathrm{a})}$ \\
Voiding diary & & & $0.045^{\mathrm{a})}$ \\
Frequency per day & $12.53 \pm 4.479$ & $16.65 \pm 7.986$ & $<0.001^{\mathrm{a})}$ \\
MVV (mL) & $244.53 \pm 84.332$ & $143.48 \pm 63.145$ & $0.438^{\mathrm{a})}$ \\
MiVV (mL) & $57.03 \pm 37.43$ & $42.61 \pm 38.283$ & $0.391^{\mathrm{b})}$ \\
Urgency & $23(71.8)$ & $14(60.9)$ & $0.383^{\mathrm{c})}$ \\
Urge incontinence & $5(15.6)$ & $1(4.3)$ & \\
\hline
\end{tabular}

Values are presented as mean \pm standard deviation or number $(\%)$.

IC/BPS, interstitial cystitis/bladder pain syndrome; MBC, maximum cystometric bladder capacity; MVV, maximal voided volume; MiVV, minimal voided volume.

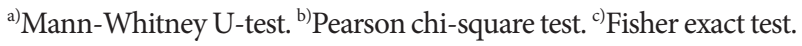


Table 2. Comparison of urodynamic parameters in IC/BPS patients with and without a Hunner lesion

\begin{tabular}{|c|c|c|c|}
\hline Variable & Non-Hunner IC/BPS ( $\mathrm{n}=32)$ & Hunner IC/BPS $(\mathrm{n}=23)$ & P-value \\
\hline \multicolumn{4}{|l|}{ Free uroflowmetry } \\
\hline $\mathrm{Qmax}(\mathrm{mL} / \mathrm{sec})$ & $15.38 \pm 6.762$ & $12.39 \pm 6.323$ & 0.130 \\
\hline Voided volume (mL) & $198.1 \pm 110.8$ & $138.5 \pm 100.3$ & $0.045^{\mathrm{a})}$ \\
\hline PVR (mL) & $30.19 \pm 33.785$ & $25.43 \pm 33.251$ & 0.607 \\
\hline \multicolumn{4}{|l|}{ Filling cystometry } \\
\hline Pdetmax $\left(\mathrm{cm} \mathrm{H}_{2} \mathrm{O}\right)$ & $9.81 \pm 4.889$ & $12.43 \pm 13.591$ & 0.384 \\
\hline DOA & $2(6.3)$ & $2(8.7)$ & 1.000 \\
\hline $\mathrm{MBC}(\mathrm{mL})$ & $286.59 \pm 69.70$ & $182.09 \pm 72.722$ & $<0.001^{*}$ \\
\hline Compliance $\left(\mathrm{mL} / \mathrm{cm} \mathrm{H}_{2} \mathrm{O}\right)$ & $30.3 \pm 9.7$ & $24.0 \pm 12.8$ & 0.035 \\
\hline First sense $(\mathrm{mL})$ & $129 \pm 74.270$ & $71 \pm 39.806$ & $0.001^{*}$ \\
\hline Normal desire (mL) & $160.44 \pm 77.947$ & $102.41 \pm 57.705$ & $0.004^{*}$ \\
\hline Strong desire $(\mathrm{mL})$ & $229.59 \pm 68.306$ & $137.45 \pm 57.495$ & $<0.001^{*}$ \\
\hline \multicolumn{4}{|l|}{ Voiding cystometry } \\
\hline PdetQmax $\left(\mathrm{cm} \mathrm{H}_{2} \mathrm{O}\right)$ & $23.42 \pm 13.58$ & $20.85 \pm 13.562$ & 0.512 \\
\hline Pdetmax $\left(\mathrm{cm} \mathrm{H}_{2} \mathrm{O}\right)$ & $37.55 \pm 17.043$ & $39.75 \pm 24.700$ & 0.708 \\
\hline $\operatorname{MUCP}\left(\mathrm{cm} \mathrm{H}_{2} \mathrm{O}\right)$ & $73.75 \pm 42.356$ & $83 \pm 16.971$ & 0.769 \\
\hline Volume discrepancy between MVV and MBC (mL) & $35.81 \pm 77.40$ & $32.08 \pm 82.87$ & 0.865 \\
\hline
\end{tabular}

Values are presented as mean \pm standard deviation or number (\%).

IC/BPS, interstitial cystitis/bladder pain syndrome; Qmax, maximal urine flow rate; PVR, postvoid residual urine volume; Pdetmax, maximal detrusor pressure; DOA, detrusor overactivity; MBC, maximum cystometric bladder capacity; PdetQmax, detrusor pressure at maximal urine flow rate; MUCP, maximal urethral closure pressure; MVV, maximal voided volume.

${ }^{*} \mathrm{P}<0.05$, statistically significant differences.
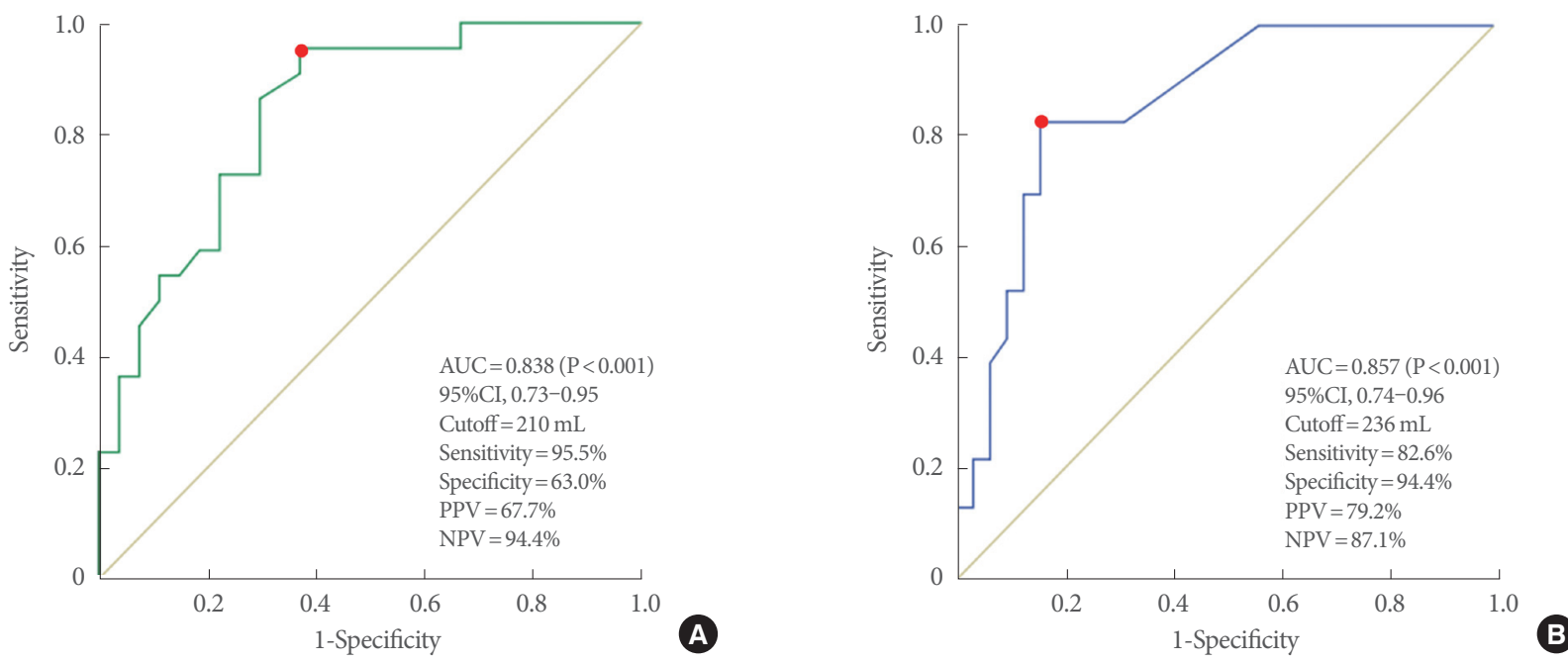

Fig. 1. The receiver operating characteristic curve analysis of SDV (A) and MBC (B). Green line, SDV; blue line, MBC; yellow line, reference line. SDV, strong desire to void; MBC, maximum cystometric bladder capacity; AUC, area under the curve; CI, confidence interval; NPV, negative predictive value; PPV, positive predictive value.

\section{DISCUSSION}

It is still unclear whether Hunner IC and non-Hunner IC are different phenotypes of the same disease condition or 2 separate disease entities [11]. However, previous studies have demonstrated differences in age $[8,12]$, bladder capacity $[12,13]$, 
histopathological and neurobiological characteristics [14], and responses to treatment according to the presence of a Hunner lesion. Consequently, some authors have attempted to characterize patients' presenting symptoms according to the presence of a Hunner lesion $[12,15]$ and Boudry et al. [16] even suggested that a voiding diary could predict the cystoscopic appearance of the bladder. Although they demonstrated a relationship between clinical data and cystoscopic findings, they relied mainly on patients' subjective symptoms. In contrast, we focused on differences in objective urodynamic parameters according to the presence of a Hunner lesion. To our knowledge, this is the first study to compare urodynamic parameters between Hunner IC/BPS and non-Hunner IC/BPS.

In our study, 23 patients (41.8\%) with IC/BPS had a Hunner lesion, which is higher than the prevalence of $10 \%-20 \%$ reported in previous studies $[17,18]$. This may reflect the current diagnostic paradigm of IC/BPS, which relies mainly on the patient's history and physical examination findings, and places less emphasis on diagnostic tests such as cystoscopy and urodynamics $[19,20]$. Nevertheless, categorizing patients into the Hunner IC/BPS and non-Hunner IC/BPS subtypes might improve our overall understanding of IC/BPS, as well as help clinicians determine whether more invasive evaluations, such as cystoscopy/hydrodistention, may be needed to rule out ulcerative disease. In fact, the problem is to determine which IC/BPS patients should undergo cystoscopy/hydrodistention, and when these procedures should be done, since the clinical findings and characteristics of the pain cannot predict the cystoscopic appearance [15].

The value of urodynamic parameters in IC/BPS is controversial [21-25]. Currently, an insufficient number of studies have discussed urodynamics and the role thereof in IC/PBS. In early studies, most IC/BPS patients showed a low volume at first sensation on filling cystometry [22,23]. Recent studies have mainly focused on the associations between symptom severity and urodynamic parameters, and have found results consistent with our findings [24,26,27]. Kirkemo et al. [26] found that the FDV and $\mathrm{MBC}$ were correlated with frequency, urgency, and nocturia as measured by voiding diaries, and Sastry et al. [24] reported that all urodynamic parameters and volumes were inversely correlated with the interstitial cystitis problem index score. Unfortunately, the previous studies that analyzed urodynamic parameters in IC/BPS patients did not investigate the associations of urodynamic parameters with different types of IC/BPS, except for Kuo and Kuo [27]. They reported that the severity of glomerulation was negatively correlated with urodynamic parameters. However, a recent systemic review cast doubt on the diagnostic usefulness of glomerulation, as it is not correlated with symptoms in patients with IC/BPS [28].

Meanwhile, considering the previous studies showing that patients with a Hunner lesion had worse urinary symptoms, it is straightforward to hypothesize that there are differences in urodynamic parameters according to the presence of a Hunner lesion. In this study, we found that the patients with Hunner IC/BPS had lower volumes measured objectively during a UDS (FDV, NDV, SDV, and MBC) than patients with non-Hunner IC/BPS. Additionally, patients with Hunner IC/BPS had lower bladder compliance than patients with non-Hunner IC/BPS. It has been found that bladder compliance in patients with IC/ BPS is normal, because the hypersensitivity to filling presents before the consequent increase in detrusor pressure can prevent the bladder from filling to the point of noncompliance [29,30]. However, in our study, the findings in patients with Hunner IC/ BPS were not consistent with those reported in previous studies, which may be due to a reduction in the elastic properties of the chronically inflamed bladder leading to a gradual increase in detrusor pressure on filling [31]. A recent review of histopathological examinations supported this proposal regarding the pathogenesis of Hunner IC/BPS, finding that Hunner IC/ BPS was associated with more severe suburothelial inflammation than non-Hunner IC/BPS [32]. Thus, these findings suggest that Hunner IC/BPS is a condition of higher grade of urothelial dysfunction than non-Hunner IC/BPS.

Additionally, we analyzed the ROC curves of urodynamic parameters and MVV through the voiding diaries. Based on the ROC analysis, the AUC values of FDV, NDV, and MVV were less than 0.80 , indicating that they did not have sufficient value as diagnostic tools. However, we determined cutoff values of the SDV and MBC capable of predicting the presence of a Hunner lesion among IC/BPS patients with relatively high specificity and sensitivity. Our findings suggest that patients with an SDV $\leq 210 \mathrm{~mL}$ or an $\mathrm{MBC} \leq 236 \mathrm{~mL}$ need a cystoscopic examination to determine whether a Hunner lesion is present. However, in agreement with the International Consultation on IC in Japan and the American Urological Association guidelines, we do not recommend routine urodynamic testing in women with IC/BPS. In our previous study, we proposed certain indications for performing a UDS in patients with IC/BPS to differentiate it from severe overactive bladder if the diagnosis is in doubt [33]. Thus, our cutoff value of the SDV and MBC 
may provide additional information to clinicians when performing urodynamic testing in certain groups of patients with IC/BPS.

A limitation of our study was the small number of patients. However, we tried to minimize the risk of extreme values by selecting patients according to a standard protocol. A future prospective study with a larger sample size is needed to further confirm our findings. An additional potential criticism is that our study was not conducted prospectively. However, our patients were evaluated according to our institutional protocol. Another potential criticism is that we did not evaluate the differences in pain characteristics between Hunner IC/BPS and non-Hunner IC/BPS. Although the evaluation of differences in pain characteristics between Hunner IC/BPS and non-Hunner IC/BPS may provide more information, doing so was outside the scope of the present study, which aimed to identify objective parametric differences. Because pain is affected by various factors, including psychological, physical, and other circumstantial factors, it is difficult to objectively measure. However, according to a previous study, the pain characteristics did not differ between Hunner IC/BPS and non-Hunner IC/BPS [15].

In conclusion, our study is significant in that the differences in patients' subjective symptoms between Hunner IC/BPS and non-Hunner IC/BPS were confirmed to correspond to differences in objective urodynamic parameters. Although a UDS is not mandatory in the routine workup for IC/BPS, we hope that our findings might provide useful information for the diagnosis and treatment of IC/BPS patients. Additionally, we suggest that UDS may play an optional diagnostic role in identifying Hunner lesion.

\section{AUTHOR CONTRIBUTION STATEMENT}

- Full access to all the data in the study and takes responsibility for the integrity of the data and the accuracy of the data analysis: Sun Tae Ahn, Mi Mi Oh

- Study concept and design: $\mathrm{Mi} \mathrm{Mi} \mathrm{Oh}$

- Acquisition of data: Hyeong Guk Jeong, Tae Yong Park

- Analysis and interpretation of data: Sun Tae Ahn, Mi Mi Oh

- Drafting of the manuscript: Sun Tae Ahn

- Critical revision of the manuscript for important intellectual content: Jong Wook Kim, Mi Mi Oh

- Statistical analysis: Sun Tae Ahn

- Administrative, technical, or material support: Jong Wook Kim, Hong Seok Park, Du Geon Moon
- Study supervision: Jeong Gu Lee, Du Geon Moon, Hong Seok Park

\section{REFERENCES}

1. Abrams P, Cardozo L, Fall M, Griffiths D, Rosier P, Ulmsten U, et al. The standardisation of terminology of lower urinary tract function: report from the Standardisation Sub-committee of the International Continence Society. Am J Obstet Gynecol 2002;187:116-26.

2. Warren JW, Meyer WA, Greenberg P, Horne L, Diggs C, Tracy JK. Using the International Continence Society's definition of painful bladder syndrome. Urology 2006;67:1138-42; discussion 1142-3.

3. Fall M, Logadottir Y, Peeker R. Interstitial cystitis is bladder pain syndrome with Hunner's lesion. Int J Urol 2014;21 Suppl 1:79-82.

4. van de Merwe JP, Nordling J, Bouchelouche P, Bouchelouche K, Cervigni M, Daha LK, et al. Diagnostic criteria, classification, and nomenclature for painful bladder syndrome/interstitial cystitis: an ESSIC proposal. Eur Urol 2008;53:60-7.

5. Ens G, Garrido GL. Role of cystoscopy and hydrodistention in the diagnosis of interstitial cystitis/bladder pain syndrome. Transl Androl Urol 2015;4:624-8.

6. Wennevik GE, Meijlink JM, Hanno P, Nordling J. The role of glomerulations in bladder pain syndrome: a review. J Urol 2016;195:1925.

7. Erickson DR, Tomaszewski JE, Kunselman AR, Bentley CM, Peters KM, Rovner ES, et al. Do the National Institute of Diabetes and Digestive and Kidney Diseases cystoscopic criteria associate with other clinical and objective features of interstitial cystitis? J Urol 2005; 173:93-7.

8. Peters KM, Killinger KA, Mounayer MH, Boura JA. Are ulcerative and nonulcerative interstitial cystitis/painful bladder syndrome 2 distinct diseases? A study of coexisting conditions. Urology 2011; 78:301-8

9. Hanno PM, Burks DA, Clemens JQ, Dmochowski RR, Erickson D, Fitzgerald MP, et al. AUA guideline for the diagnosis and treatment of interstitial cystitis/bladder pain syndrome. J Urol 2011;185:216270.

10. Fluss R, Faraggi D, Reiser B. Estimation of the Youden Index and its associated cutoff point. Biom J 2005;47:458-72.

11. Yoshimura N. Editorial comment from Dr. Yoshimura to Interstitial cystitis is bladder pain syndrome with Hunner's lesion. Int J Urol 2014;21 Suppl 1:83-4.

12. Braunstein R, Shapiro E, Kaye J, Moldwin R. The role of cystoscopy in the diagnosis of Hunner's ulcer disease. J Urol 2008;180:1383-6.

13. Koziol JA, Adams HP, Frutos A. Discrimination between the ulcerous and the nonulcerous forms of interstitial cystitis by noninvasive 
findings. J Urol 1996;155:87-90.

14. Peeker R, Fall M. Toward a precise definition of interstitial cystitis: further evidence of differences in classic and nonulcer disease. J Urol 2002;167:2470-2.

15. Killinger KA, Boura JA, Peters KM. Pain in interstitial cystitis/bladder pain syndrome: do characteristics differ in ulcerative and nonulcerative subtypes? Int Urogynecol J 2013;24:1295-301.

16. Boudry G, Labat JJ, Riant T, Le Normand L, Manunta A, Bensalah $\mathrm{K}$, et al. Validation of voiding diary for stratification of bladder pain syndrome according to the presence/absence of cystoscopic abnormalities: a two-centre prospective study. BJU Int 2013;112:E164-8.

17. Grégoire M, Liandier F, Naud A, Lacombe L, Fradet Y. Does the potassium stimulation test predict cystometric, cystoscopic outcome in interstitial cystitis? J Urol 2002;168:556-7.

18. Hanno PM, Landis JR, Matthews-Cook Y, Kusek J, Nyberg L Jr. The diagnosis of interstitial cystitis revisited: lessons learned from the National Institutes of Health Interstitial Cystitis Database study. J Urol 1999;161:553-7.

19. Evans RJ, Sant GR. Current diagnosis of interstitial cystitis: an evolving paradigm. Urology 2007;69(4 Suppl):64-72.

20. Kim HJ. Update on the pathology and diagnosis of interstitial cystitis/bladder pain syndrome: a review. Int Neurourol J 2016;20:13-7.

21. Kuo HC. Urodynamic study and potassium sensitivity test for women with frequency-urgency syndrome and interstitial cystitis. Urol Int 2003;71:61-5.

22. Nigro DA, Wein AJ, Foy M, Parsons CL, Williams M, Nyberg LM Jr, et al. Associations among cystoscopic and urodynamic findings for women enrolled in the Interstitial Cystitis Data Base (ICDB) Study. Urology 1997;49(5A Suppl):86-92.

23. Perez-Marrero R, Emerson L, Juma S. Urodynamic studies in interstitial cystitis. Urology 1987;29(4 Suppl):27-30.
24. Sastry DN, Hunter KM, Whitmore KE. Urodynamic testing and interstitial cystitis/painful bladder syndrome. Int Urogynecol J 2010; 21:157-61.

25. Steinkohl WB, Leach GE. Urodynamic findings in interstitial cystitis. Urology 1989;34:399-401.

26. Kirkemo A, Peabody M, Diokno AC, Afanasyev A, Nyberg LM Jr, Landis JR, et al. Associations among urodynamic findings and symptoms in women enrolled in the Interstitial Cystitis Data Base (ICDB) Study. Urology 1997;49(5A Suppl):76-80.

27. Kuo YC, Kuo HC. The urodynamic characteristics and prognostic factors of patients with interstitial cystitis/bladder pain syndrome. Int J Clin Pract 2013;67:863-9.

28. Doiron RC, Tolls V, Irvine-Bird K, Kelly KL, Nickel JC. Clinical phenotyping does not differentiate Hunner lesion subtype of interstitial cystitis/bladder pain syndrome: a relook at the role of cystoscopy. J Urol 2016;196:1136-40.

29. Rovner ES, Wein AJ. Once-daily, extended-release formulations of antimuscarinic agents in the treatment of overactive bladder: a review. Eur Urol 2002;41:6-14.

30. Siroky MB. Is it interstitial cystitis? Diagnostic distinctions in reduced bladder capacity. Contemp Urol 1994;6:13-22.

31. Offiah I, McMahon SB, O’Reilly BA. Interstitial cystitis/bladder pain syndrome: diagnosis and management. Int Urogynecol J 2013; 24:1243-56.

32. Jhang JF, Kuo HC. Pathomechanism of interstitial cystitis/bladder pain syndrome and mapping the heterogeneity of disease. Int Neurourol J 2016;20(Suppl 2):S95-104.

33. Shim JS, Kang SG, Park JY, Bae JH, Kang SH, Park HS, et al. Differences in urodynamic parameters between women with interstitial cystitis and/or bladder pain syndrome and severe overactive bladder. Urology 2016;94:64-9. 